\title{
A Professional Development Model for Medical Laboratory Scientists Working in the Microbiology Laboratory
}

\author{
MEGAN H. AMERSON, LILA PULIDO, MELINDA N. GARZA, FAHEEM A. ALI, BRANDY \\ GREENHILL, CHRISTOPHER L. EINSPAHR, JOSEPH YARSA, PRAMILLA K. SOOD, PETER C. HU
}

\section{ABSTRACT}

The University of Texas M.D. Anderson Cancer Center, Division of Pathology and Laboratory Medicine is committed to providing the best pathology and medicine through: state-of-the art techniques, progressive ground-breaking research, education and training for the clinical diagnosis and research of cancer and related diseases. After surveying the laboratory staff and other hospital professionals, the Department administrators and Human Resource generalists developed a professional development model for Microbiology to support laboratory skills, behavior, certification, and continual education within its staff. This model sets high standards for the laboratory professionals to allow the labs to work at their fullest potential; it provides organization to training technologists based on complete laboratory needs instead of training technologists in individual areas in which more training is required if the laboratory needs them to work in other areas. This model is a working example for all microbiology based laboratories who want to set high standards and want their staff to be acknowledged for demonstrated excellence and professional development in the laboratory. The PDM model is designed to focus on the needs of the laboratory as well as the laboratory professionals.

ABBREVIATIONS: PDM, professional development model; UTMDACC, University of Texas MD Anderson Cancer Center

INDEX TERMS: Microbiology, Professional, Laboratory, Clinical, Model

Clin Lab Sci 2012;25(2):74

Megan H. Amerson, School of Health Professions, The University of Texas, MD Anderson Cancer Center,
Houston, TX 77030

Lila Pulido, Division of Pathology and Laboratory Medicine, The University of Texas, MD Anderson Cancer Center, Houston, TX 77030

Melinda N. Garza, School of Health Professions, The University of Texas, MD Anderson Cancer Center, Houston, TX 77030

Fabeem A. Ali, School of Health Professions, The University of Texas, MD Anderson Cancer Center, Houston, TX 77030

Brandy Greenhill, School of Health Professions, The University of Texas, MD Anderson Cancer Center, Houston, TX 77030

Christopher L. Einspahr, School of Health Professions, The University of Texas, MD Anderson Cancer Center, Houston, TX 77030

Joseph Yarsa, Division of Pathology and Laboratory Medicine, The University of Texas, MD Anderson Cancer Center, Houston, TX 77030

Pramilla K. Sood, Division of Pathology and Laboratory Medicine, The University of Texas, MD Anderson Cancer Center, Houston, TX 77030

Peter C. Hu, PhD, School of Health Professions, The University of Texas, MD Anderson Cancer Center, Houston, TX 77030

Address for Correspondence: Peter Hu, PhD, 1515 Holcombe Blvd, Unit 2, Houston, TX 77030, 713-5633095,pchu@mdanderson.org 


\section{CLINICAL PRACTICE}

\section{INTRODUCTION}

The PDM consists of four competency levels: Discovery, Application, Maturation, and Expert (Figures 2-5). A person's competency is defined as their ability to integrate their knowledge, attitudes, skills, behaviors, and standards of practice into their actual performance. $^{1,2}$ Each competency level of the PDM requires Medical Technologist to learn and become proficient at the designated work stations (Figure 1), and master the skill set through experience and practice.

\begin{tabular}{|l|}
\hline Routine/Vitek/E-Test/Gram Stain (12 wks) \\
Anaerobes ( 4 wks) \\
Blood Smears/IC (4 wks) \\
Blood Cultures/Vitek/E-Test/Gram Stain (12 wks) \\
Late Shift Responsibilities (5 wks) \\
Mycology ( 8 wks) \\
Virology (16 wks) \\
AFB (6 wks) \\
Serology (4 wks)
\end{tabular}

Figure 1. Stations considered for training and rotations of laboratory personnel

The PDM creates for a schedule for laboratory personnel to be trained in the fundamental skills through the advanced application, projects and education. Each level is designed to build on the knowledge they acquired from the previous levels. Continuing education and advanced certification also allow for opportunities for progression in the laboratory professionals career outside of the PDM. The PDM makes progression in the laboratory organized, planned and easy to follow from both a managerial and technical position as well as motivational for the participants who complete the program.

\section{Progression through the Model}

The Model consists of four levels: Discovery, Application, Maturation, and Expert. Technologists are required to complete a level before proceeding to the next. Technologists are also evaluated by the laboratory administration before movement to the next level, and if not completed in the expected time the technologist is evaluated on the cause of the slowed progression in the laboratory and in their professional behavior. The model is based on the skills, certification, and education needed by the lab on the business and technical levels as well as the demonstrated expertise and professional interests. It is designed to encourage teamwork and not competitiveness since everyone must complete mastery of the same objectives in the same time frame. Continuing education opportunities provided to the participants are available in various ways: support for attending conferences (local, state, national, or even international), laboratory monthly or weekly in-services, institutional weekly in-services, or advanced education such as Masters or PhD programs. At UTMDACC, we offer tuition assistance for those who wish to continue their education. With the ease of access to many on-line accredited education programs, laboratory professionals are able to take courses without sacrificing their existing work schedule.

Each level has a set time frame in which completion is encouraged for Technologists to develop and master skills and work in a timely manner. Adjustments to the time frames may be made based on previous experience such as if a Technologist was to leave MDACC but then return to the staff at a later point in their professional career, or if a professional has demonstrated mastery of a level the time frame may be altered with approval of the laboratory administration.

All new medical laboratory scientists hired at MDACC are given the opportunity to enroll in the PDM. Thus, eligibility requirements are basically those set by the HR department during the initial hiring process. Existing employees at the time of the PDM rollout were also given the opportunity to enroll.

Table 1 shows the minimum-maximum completion times for each level. Mastery of each level must be completed in the appropriate time scheme shown. Failure to complete each level within the maximum time frame can lead to disciplinary action up to and including termination. Once a technologist has completed the Maturation level the technologist receives a new title of Senior Technologist. Completion of the Expert level is optional and is based on two different tracks at the bench or laboratory management depending on the current position that the scientist holds and the capability and interests of the individual and needs of the laboratory. Employees who were previously employed by MDACC before the models were set into place may opt out of the PDM, but are not subjects to raises based on the model.

The new employee will meet with the laboratory manager to determine the timeline for progression and 
discuss their personalized training through the model. There are nine different sections of the microbiology laboratory that the technologist can work through: Routine, Blood Smear, Blood culture, Late Shift, Anaerobe, Mycology, Virology, AFB, and Serology. The model allows for effective education in management of work flow related to the laboratory and is critical to its success.

Table 1. Set Time Spans for Each Competency Level

\begin{tabular}{ccc}
\hline Level & Minimum Time & Maximum Time \\
\hline Discovery & 12 months & 18 months \\
Application & 18 months & 24 months \\
Maturation & 18 months & 24 months \\
Expert & N/A & N/A \\
\hline
\end{tabular}

Once training has begun in Discovery, the technologist is trained in three areas: Routine, Blood Smear, Blood Culture and/or Virology that make up the majority of the lab general work. It is up to the technologist to complete the mastery of these sections and the education and competency required at this level and all of the other levels through Maturation in the appropriate time frame. Once in Maturation the laboratory manager and technologist will decide on a specialty level to be trained in based on interest of the technologist and laboratory business needs. Research and projects assigned to technologists in each specific level are based on the same criteria, interest and business needs. Below is an outline of the PDM used in the micro lab (See Figures 2-5).

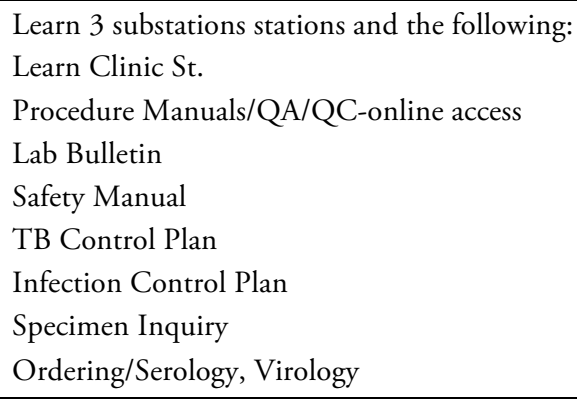

Figure 2. Criteria for advancement through the discovery phase

\section{Compensation for Completion of Levels}

Once a technologist has completed a level they are evaluated. Compensation is given by an increase in salary and a satisfaction of completion on each level. Salary compensation is regulated by the institution and the Human Resource department. Technologists say that while the salary benefit is perk to their job the greatest satisfaction comes from being able to express their interests and increase their learning and development of their skills in their area of expertise with continual encouragement.

Learn 1 additional station and:

Tech learns new material, build on existing foundations, and rotating through the stations that one has learned.

Maintain competency in areas that the tech has been trained.

Tech teaches students and employees.

Tech does minor projects.

Tech gets involved with student projects and develops techniques to manage projects.

Figure 3. Criteria for advancement through the application phase

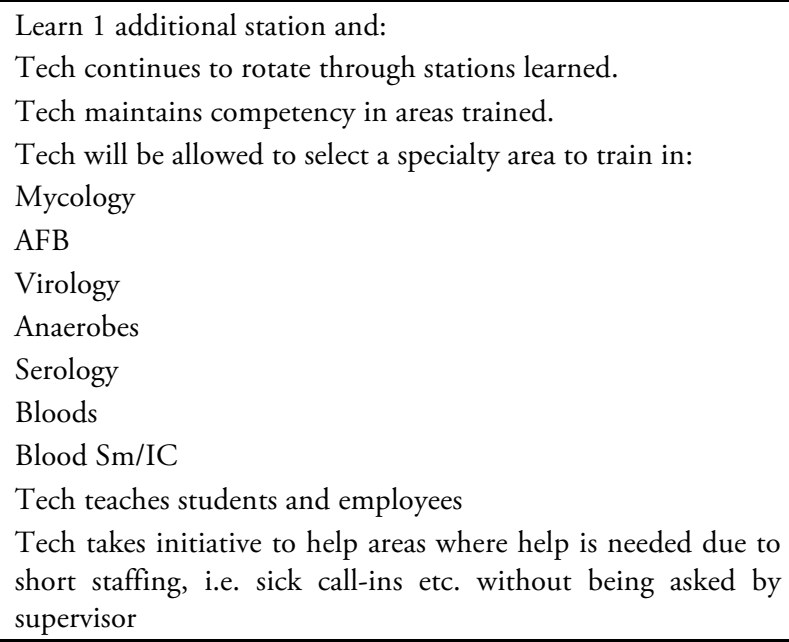

Tech takes initiative to help areas where help is needed due to short staffing, i.e. sick call-ins etc. without being asked by supervisor

Figure 4. Criteria for advancement through the maturation phase.

\section{CONCLUSION}

The professional development model designed for Microbiology was the first implemented at UTMDACC. It was designed to promote professional growth, enthusiasm, and fulfillment and acknowledge professional development of medical laboratory scientists as they progress in their field. While progression "up the ladder" can be difficult or frustrating in some settings, this model serves as an ideal example to emulate for microbiology laboratories who wish to establish a framework for organizing education, encouraging professional enthusiasm and fulfillment and promoting professional growth of their laboratory professionals. It is helpful to inform laboratory professionals that continual growth and learning is 


\section{CLINICAL PRACTICE}

expected and necessary for advancement in laboratory professions. The model is an ideal example for other microbiology laboratories who wish to achieve organization in the training of their laboratory professionals and to promote professional growth.

\footnotetext{
Tech continues to rotate through stations learned.

Tech maintains competency in areas trained.

Tech gets involved with developments of tests and projects.

Tech learns to manage inventory.

Tech learns to write and review procedures.

Tech assists Senior Tech with trouble shooting of instruments.

Tech teaches students and employees.

Tech assists Senior Tech with Competency testing of employees in areas trained.

Takes the initiative to help areas where help is needed due to short staffing, sick call-ins etc. without being asked by a Supervisor.

Tech helps with validation testing.

Option to get Specialty certification.

Tech helps with equipment inventory on yearly basis.

Tech participates in interim CAP inspections or external CAP inspections.

Tech participates in training and management course.

Tech has the choice to take technical track or Supervisory track.
}

Figure 5. Criteria for mastery of the expert phase and advancement into supervisor position
This article was supported in part by the National Institutes of Health through MD Anderson's Cancer Center Support Grant CA016672

\section{REFERENCES}

1. Lennon PA, Lovshe D, Deleon E, Reynolds A, Pulido L, Cheong S, et al. Professional development model for cytogenetic technologists at The University of Texas MD Anderson Cancer Center sets high standards. J Assoc Genet Technol. 2010;36:195-7.

2. Lennon PA, Deleon E, Reynolds A, Pulido L, Lewing C, Mehta $\mathrm{P}$, et al. A model for rewarding professional growth in the diagnostic molecular oncology laboratory. J Assoc Genet Technol. 2011;37:80-3.

3. Bruns DE. Improving training in laboratory medicine. Clin Chim Acta. 2008;393:3-4.

The peer-reviewed Clinical Practice Section seeks to publish case studies, reports, and articles that are immediately useful, are of a practical nature, or contain information that could lead to improvement in the quality of the clinical laboratory's contribution to patient care, including brief reviews of books, computer programs, audiovisual materials, or other materials of interest to readers. Direct all inquiries to Perry Scanlan, PhD, MT(ASCP), Medical Technology, Austin Peay State University, Room D212, Sundquist Science Complex, Box 4668, Clarksville TN 37044. Clinical Laboratory Science encourages readers to respond with thoughts, questions, or comments regarding these articles. Email responses to westminsterpublishers@comcast.net. In the subject line, please type the journal issue and lead author such as "CLIN LAB SCI 25(2) RE AMERSON". Selected responses may appear in the Dialogue and Discussion section in a future issue. Responses may be edited for length and clarity. We look forward to hearing from you. 